\title{
Erratum: integrand reduction of one-loop scattering amplitudes through Laurent series expansion
}

\author{
Pierpaolo Mastrolia,,$^{a, b}$ Edoardo Mirabella $^{a}$ and Tiziano Peraro ${ }^{a}$ \\ ${ }^{a}$ Max-Planck-Institut für Physik, \\ Föhringer Ring 6, D-80805 München, Germany \\ ${ }^{b}$ Dipartimento di Fisica e Astronomia, Università di Padova, and INFN Sezione di Padova, \\ via Marzolo 8, 35131 Padova, Italy \\ E-mail: pierpaolo.mastrolia@cern.ch, mirabell@mppmu.mpg.de, \\ peraro@mppmu.mpg.de
}

ERRATUM TO: JHEP06(2012)095

ABSTRACT: We correct an error affecting eq. (6.11) of the article.

The contribution to the (integrated) amplitude $\delta \mathcal{A}_{n}$, coming from the additional MI's that arise in the higher-rank case $(r \leq n+1)$, which was given in eq. (6.11), should be replaced by,

$$
\begin{aligned}
\delta \mathcal{A}_{n}= & \sum_{i<j<k}^{n-1} c_{3,14}^{(i j k)} I_{i j k}\left[\mu^{4}\right] \\
& +\sum_{i<j}^{n-1}\left\{c_{2,13}^{(i j)} I_{i j}\left[\left(\left(q+p_{i}\right) \cdot e_{2}\right)^{3}\right]+c_{2,10}^{(i j)} I_{i j}\left[\mu^{2}\left(\left(q+p_{i}\right) \cdot e_{2}\right)\right]\right\} \\
& +\sum_{i}^{n-1}\left\{c_{1,14}^{(i)} I_{i}\left[\mu^{2}\right]+c_{1,15}^{(i)} I_{i}\left[\left(\left(q+p_{i}\right) \cdot e_{3}\right)\left(\left(q+p_{i}\right) \cdot e_{4}\right)\right]\right\} .
\end{aligned}
$$

The expression of the integral $I_{i}\left[\left(\left(q+p_{i}\right) \cdot e_{3}\right)\left(\left(q+p_{i}\right) \cdot e_{4}\right)\right]$ can be obtained by contracting the covariant decomposition (B.3) with $e_{3}^{\mu} e_{4}^{\nu}$, and by using the relation $e_{3} \cdot e_{4}=-1$. The outcome is

$$
\begin{aligned}
\left.I_{i}\left[\left(q+p_{i}\right) \cdot e_{3}\right)\left(\left(q+p_{i}\right) \cdot e_{4}\right)\right] & =\int d^{d} \bar{q} \frac{\left.\left(q+p_{i}\right) \cdot e_{3}\right)\left(\left(q+p_{i}\right) \cdot e_{4}\right)}{D_{i}}=\left(e_{3} \cdot e_{4}\right) A_{00} \\
& =-\frac{m_{i}^{2} I_{i}+I_{i}\left[\mu^{2}\right]}{4}+\mathcal{O}(\epsilon) .
\end{aligned}
$$

